\title{
Global Dynamics for a Novel Differential Infectivity Epidemic Model with Stage Structure
}

\author{
Yunguo Jin ${ }^{1,2}$ \\ ${ }^{1}$ School of Statistics, Chengdu University of Information Technology, Chengdu, Sichuan 610103, China \\ ${ }^{2}$ Key Laboratory of Statistical Information Technology and Data Mining, State Statistics Bureau, Chengdu, China \\ Correspondence should be addressed to Yunguo Jin; yunguoj@163.com
}

Received 1 September 2016; Revised 17 November 2016; Accepted 24 November 2016

Academic Editor: Zhengqiu Zhang

Copyright (c) 2016 Yunguo Jin. This is an open access article distributed under the Creative Commons Attribution License, which permits unrestricted use, distribution, and reproduction in any medium, provided the original work is properly cited.

\begin{abstract}
A novel differential infectivity epidemic model with stage structure is formulated and studied. Under biological motivation, the stability of equilibria is investigated by the global Lyapunov functions. Some novel techniques are applied to the global dynamics analysis for the differential infectivity epidemic model. Uniform persistence and the sharp threshold dynamics are established; that is, the reproduction number determines the global dynamics of the system. Finally, numerical simulations are given to illustrate the main theoretical results.
\end{abstract}

\section{Introduction}

Mathematical model that reflects the characteristics of an epidemic to some extent can help us to understand better how the disease spreads in the community and can investigate how changes in the various assumptions and parameter values affect the course of epidemic. In [1], Hyman et al. proposed a differential infectivity model that accounted for differences in infectiousness between individuals during the chronic stages and the correlation between viral loads and rates of developing AIDS. They assumed that the susceptible population was homogeneous and neglected variations in susceptibility, risk behavior, and many other factors associated with the dynamics of HIV spread. Ma et al. [2] presented several differential infectivity epidemic models under different assumptions.

In the real world, some epidemics, such as malaria, dengue, fever, gonorrhea, and bacterial infections, may have a different ability to transmit the infections in different ages. For example, measles and varicella always occur in juveniles, while it is reasonable to consider the disease transmission in adult population such as typhus and diphtheria. In recent years, epidemic models with stage structure have been studied in many papers [3-10].

In this paper, we formulate a differential infectivity epidemic model with stage structure. The proof of global stability of the endemic equilibrium utilizes a graphtheoretical approach [11-22] to the method of Lyapunov functions. Let $S_{1}$ and $S_{2}$ denote the immature susceptible and mature susceptible populations, respectively. The infectious population $I$ was subdivided into $n$ subgroups $I_{1}, I_{2}, \ldots, I_{n}$. $p_{1 k}$ and $p_{2 k}$ denote the probabilities of an immature infectious individual and a mature infectious individual enter subgroup $k$, respectively, where $\sum_{k=1}^{n} p_{1 k}=\sum_{k=1}^{n} p_{2 k}=1$. The disease incidence in the $k$ th subgroup can be calculated as $\sum_{i=1}^{2} p_{i k} \sum_{j=1}^{n} \beta_{i j} S_{i} G_{j}\left(I_{j}\right)$, where $\beta_{i j}$ is the transmission coefficient between compartments $S_{i}$ and $I_{j}$. $G_{j}\left(I_{j}\right)$ includes some special incidence functions in the literature. For instance, $G_{j}\left(I_{j}\right)=I_{j} /\left(1+\alpha_{j} I_{j}\right)$ (saturation effect). Since we do not assume that recovered individuals return into the susceptible class, the recovered class does not need to be explicitly modeled. Then, we obtain the following model:

$$
\begin{aligned}
& \dot{S_{1}}=\varphi\left(S_{1}\right)-\sum_{j=1}^{n} \beta_{1 j} S_{1} G_{j}\left(I_{j}\right)-a S_{1}, \\
& \dot{S_{2}}=a S_{1}-\sum_{j=1}^{n} \beta_{2 j} S_{2} G_{j}\left(I_{j}\right)-d_{2} S_{2}, \\
& \dot{I_{k}}=\sum_{i=1}^{2} p_{i k} \sum_{j=1}^{n} \beta_{i j} S_{i} G_{j}\left(I_{j}\right)-m_{k} I_{k}, \quad k=1,2, \ldots, n,
\end{aligned}
$$


where $\varphi\left(S_{1}\right)=b-d_{1} S_{1}$ with $b$ being the recruitment constant and $d_{1}$ being the natural death rate. $a$ is the conversion rate from an immature individual to a mature individual. $d_{2}$ is the natural death rate of the mature susceptible class. $m_{k}=d_{k}^{I}+$ $\gamma_{k}$, where $d_{k}^{I}$ is the death rate of $I$ population in subgroup $k$ and $\gamma_{k}$ is the recovery rate in the $k$ th subgroup. All parameter values are assumed to be nonnegative and $b, a, m_{k}, d_{1}, d_{2}>0$.

The organization of this paper is as follows. In Section 2, we prove some preliminary results for system (1). In Section 3, the main theorem of this paper is stated and proved. In Section 4, numerical simulations which support our theoretical analysis are given.

\section{Preliminaries}

We assume the following.

(A1) $G_{k}$ is continuous and Lipschitz on $[0,+\infty), G_{k}(x) / x$ is nonincreasing on $(0,+\infty)$, and

$$
\delta_{k}=\lim _{x \rightarrow 0} \frac{G_{k}(x)}{x}>0 \text { exists. }
$$

From our assumptions, it is clear that system (1) has a unique solution for any given initial data $\left(S_{1}(0), S_{2}(0)\right.$, $\left.I_{1}(0), \ldots, I_{n}(0)\right)$ with $S_{1}(0)>0, S_{2}(0)>0$, and $I_{k}(0)>0$ for $k=1,2, \ldots, n$ and the solution remains nonnegative. We see that system (1) exits in a disease-free equilibrium $P_{0}=$ $\left(S_{1}^{0}, S_{2}^{0}, 0, \ldots, 0\right)$, where $S_{2}^{0}=a S_{1}^{0} / d_{2}$. Let $\underline{m}=\min \left\{d_{1}, d_{2}\right.$, $\left.m_{1}, \ldots, m_{n}\right\}$. Then, we derive from (1) that the region,

$$
\begin{aligned}
\Gamma & =\left\{\left(S_{1}, S_{2}, I_{1}, \ldots, I_{n}\right) \in \mathbb{R}_{+}^{n+2}: S_{1} \leq S_{1}^{0}, S_{2} \leq S_{2}^{0}, S_{1}\right. \\
& \left.+S_{2}+\sum_{k=1}^{n} I_{k} \leq \frac{b}{\underline{m}}\right\},
\end{aligned}
$$

is a forward invariant compact absorbing set with respect to (1). Also let $\Gamma^{\circ}$ denote the interior of $\Gamma$. The next generation matrix for system (1) is

$$
\begin{aligned}
\mathbf{Q} & :=\left[\frac{\sum_{i=1}^{2} p_{i k} \beta_{i j} S_{i}^{0} \delta_{j}}{m_{k}}\right]_{n \times n} \\
& =\left[\begin{array}{ccc}
\frac{\sum_{i=1}^{2} p_{i 1} \beta_{i 1} S_{i}^{0} \delta_{1}}{m_{1}} & \cdots & \frac{\sum_{i=1}^{2} p_{i 1} \beta_{i n} S_{i}^{0} \delta_{n}}{m_{1}} \\
\vdots & \ddots & \vdots \\
\frac{\sum_{i=1}^{2} p_{i n} \beta_{i 1} S_{i}^{0} \delta_{1}}{m_{n}} & \cdots & \frac{\sum_{i=1}^{2} p_{i n} \beta_{i n} S_{i}^{0} \delta_{n}}{m_{n}}
\end{array}\right] .
\end{aligned}
$$

Then, we define the basic reproduction number as the spectral radius of $Q, R_{0}=\rho(\mathbf{Q})$. A square matrix is said to be reducible, if there is a permutation matrix $P$, such that $P^{\prime} A P$ is a block upper triangular matrix; otherwise it is irreducible.

\section{Main Results}

In the section, we will study the global asymptotical stability of equilibria of system (1).
Theorem 1. Assume that (A1) holds and $\mathbf{B}=\left[\sum_{i=1}^{2} p_{i k} \beta_{i j}\right]$ is irreducible.

(1) If $R_{0} \leq 1$, then $P_{0}$ is globally asymptotically stable in $\Gamma$.

(2) If $R_{0}>1$, then $P_{0}$ is unstable and system (1) admits at least one endemic equilibrium in $\Gamma^{\circ}$.

Proof. Let $\mathbf{S}=\left(S_{1}, S_{2}\right), \mathbf{S}^{\mathbf{0}}=\left(S_{1}^{0}, S_{2}^{0}\right), \mathbf{I}=\left(I_{1}, I_{2}, \ldots, I_{n}\right)$, and $\mathbf{Q}(\mathbf{S}, \mathbf{I})=\left(\sum_{i=1}^{2}\left(p_{i k} \beta_{i j} S_{i} G_{j}\left(I_{j}\right)\right) / m_{k} I_{j}\right)_{n \times n}$. Notice that $\mathbf{B}$ is irreducible, and then $\mathbf{Q}$ is also irreducible. Hence, there exists $\omega_{k}>0, k=1,2, \ldots, n$, such that

$$
\left(\omega_{1}, \omega_{2}, \ldots, \omega_{n}\right) \rho(\mathbf{Q})=\left(\omega_{1}, \omega_{2}, \ldots, \omega_{n}\right) \mathbf{Q} .
$$

Define $L=\sum_{k=1}^{n}\left(\omega_{k} I_{k} / m_{k}\right)$. Then

$$
\begin{aligned}
\dot{L} & =\sum_{k=1}^{n} \omega_{k}\left[\frac{\sum_{i=1}^{2} p_{i k} \sum_{j=1}^{n} \beta_{i j} S_{i} G_{j}\left(I_{j}\right)}{m_{k}}-I_{k}\right] \\
& =\left(\omega_{1}, \omega_{2}, \ldots, \omega_{n}\right)\left[\mathbf{Q}(\mathbf{S}, \mathbf{I}) \mathbf{I}^{T}-\mathbf{I}^{T}\right] \\
& \leq\left(\omega_{1}, \omega_{2}, \ldots, \omega_{n}\right)\left[\mathbf{Q} \mathbf{I}^{T}-\mathbf{I}^{T}\right] \\
& =[\rho(\mathbf{Q})-1]\left(\omega_{1}, \omega_{2}, \ldots, \omega_{n}\right) \mathbf{I}^{T} .
\end{aligned}
$$

We see that the only compact invariant subset of the set where $\dot{L}=0$ is the singleton $\left\{P_{0}\right\}$. By LaSalle's Invariance Principle, $P_{0}$ is globally asymptotically stable in $\Gamma$ if $R_{0} \leq 1$.

If $R_{0}>1$, by continuity, we obtain that $\dot{L}=\left(\omega_{1}, \omega_{2}, \ldots\right.$, $\left.\omega_{n}\right)\left[\mathbf{Q}(\mathbf{S}, \mathbf{I}) \mathbf{I}^{T}-\mathbf{I}^{T}\right]>0$ in a neighborhood of $P_{0}$ in $\Gamma^{\circ}$. This implies that $P_{0}$ is unstable. From a uniform persistence result of [23] and a similar argument as in the proof of Proposition 3.3 of [24], we can deduce that the instability of $P_{0}$ implies the uniform persistence of system (1) in $\Gamma^{\circ}$. This together with the uniform boundedness of solutions of system (1) in $\Gamma^{\circ}$ implies that system (1) has an endemic equilibrium in $\Gamma^{\circ}$ (see Theorem 2.8.6 of [25] or Theorem D.3 of [26]). The proof is completed.

By Theorem 1, we have the idea that if $\mathbf{B}=\left[\sum_{i=1}^{2} p_{i k} \beta_{i j}\right]$ is irreducible, (A1) holds and $R_{0}>1$, and then system (1) exists in endemic equilibrium $P^{*}$ in $\Gamma^{\circ}$. Let $P^{*}=\left(S_{1}^{*}, S_{2}^{*}, I_{1}^{*}, \ldots, I_{n}^{*}\right)$, and then the components of $P^{*}$ satisfy

$$
\begin{gathered}
\varphi\left(S_{1}^{*}\right)=\sum_{i=1}^{2} \sum_{j=1}^{n} \beta_{i j} S_{i}^{*} G_{j}\left(I_{j}^{*}\right)+d_{2} S_{2}^{*}, \\
a S_{1}^{*}=\sum_{j=1}^{n} \beta_{2 j} S_{2}^{*} G_{j}\left(I_{j}^{*}\right)+d_{2} S_{2}^{*},
\end{gathered}
$$

$$
\sum_{i=1}^{2} p_{i k} \sum_{j=1}^{n} \beta_{i j} S_{i}^{*} G_{j}\left(I_{j}^{*}\right)=m_{k} I_{k}^{*} .
$$

Since $\varphi$ is strictly decreasing on $[0,+\infty)$, we have

$$
\left[\varphi\left(S_{1}\right)-\varphi\left(S_{1}^{*}\right)\right]\left(p_{1 k}-\frac{p_{1 k} S_{1}^{*}}{S_{1}}\right) \leq 0 .
$$


For convenience of notations, set

$$
\begin{aligned}
\bar{\beta}_{k j}= & \sum_{i=1}^{2} p_{i k} \beta_{i j} S_{i}^{*} G_{j}\left(I_{j}^{*}\right), \quad 1 \leq k, j \leq n, \\
\bar{B} & =\left[\begin{array}{cccc}
\sum_{l \neq 1} \bar{\beta}_{1 l} & -\bar{\beta}_{21} & \cdots & -\bar{\beta}_{n 1} \\
-\bar{\beta}_{12} & \sum_{l \neq 2} \bar{\beta}_{2 l} & \cdots & -\bar{\beta}_{n 2} \\
\vdots & \vdots & \ddots & \vdots \\
-\bar{\beta}_{1 n} & -\bar{\beta}_{2 n} & \cdots & \sum_{l \neq n} \bar{\beta}_{n l}
\end{array}\right] .
\end{aligned}
$$

Then, $\bar{B}$ is also irreducible. It follows from Lemma 2.1 of [11] that the solution space of linear system,

$$
\bar{B} \mathbf{v}=0 \text {, }
$$

has dimension 1 , with a basis

$$
\mathbf{v}:=\left(v_{1}, v_{2}, \ldots, v_{n}\right)^{T}=\left(c_{1}, c_{2}, \ldots, c_{n}\right)^{T},
$$

where $c_{k}$ denotes the cofactor of the $k$ th diagonal entry of $\bar{B}$. Note that from (12) we have

$$
\sum_{j=1}^{n} \bar{B}_{k j} v_{k}=\sum_{j=1}^{n} \bar{B}_{j k} v_{j}, \quad k=1,2, \ldots, n .
$$

From (14), we have

$$
\begin{aligned}
& \sum_{k=1}^{n} v_{k} \sum_{j=1}^{n} \sum_{i=1}^{2} p_{i k} \beta_{i j} S_{i}^{*} G_{j}\left(I_{j}\right)=\sum_{k, j=1}^{n} \sum_{i=1}^{2} p_{i j} \beta_{i k} S_{i}^{*} v_{j} G_{k}\left(I_{k}\right) \\
& =\sum_{k=1}^{n}\left[\sum_{j=1}^{n} \sum_{i=1}^{2} p_{i j} \beta_{i k} S_{i}^{*} G_{k}\left(I_{k}^{*}\right) v_{j}\right] \frac{G_{k}\left(I_{k}\right)}{G_{k}\left(I_{k}^{*}\right)} \\
& =\sum_{k=1}^{n}\left[\sum_{j=1}^{n}\left(\bar{\beta}_{j k} v_{j}\right)\right] \frac{G_{k}\left(I_{k}\right)}{G_{k}\left(I_{k}^{*}\right)} \\
& =\sum_{k=1}^{n}\left[\sum_{j=1}^{n}\left(\bar{\beta}_{k j} v_{k}\right)\right] \frac{G_{k}\left(I_{k}\right)}{G_{k}\left(I_{k}^{*}\right)} \\
& =\sum_{k=1}^{n} v_{k} \sum_{j=1}^{n} \sum_{i=1}^{2} p_{i k} \beta_{i j} S_{i}^{*} G_{j}\left(I_{j}^{*}\right) \frac{G_{k}\left(I_{k}\right)}{G_{k}\left(I_{k}^{*}\right)}
\end{aligned}
$$

We further make the following assumption.

(A2) $G_{k}$ is strictly increasing on $[0,+\infty)$, and

$$
\frac{G_{k}\left(x_{k}\right) I_{k}}{G_{k}\left(I_{k}\right) x_{k}}+\frac{G_{k}\left(I_{k}\right)}{G_{k}\left(x_{k}\right)}-\frac{I_{k}}{x_{k}} \leq 1, \quad k=1,2, \ldots, n,
$$

where $x_{k}>0$ is chosen in an arbitrary way and equality holds if $I_{k}=x_{k}$.

Theorem 2. Assume that (A1) and (A2) hold, $\sum_{k=1}^{n} v_{k}\left(p_{1 k}-\right.$ $\left.p_{2 k}\right) \geq 0$, and $\mathbf{B}=\left[\sum_{i=1}^{2} p_{i k} \beta_{i j}\right]$ is irreducible. If $R_{0}>1$, then $P^{*}$ is globally asymptotically stable in $\Gamma^{\circ}$ and thus is the unique endemic equilibrium.
Proof. Consider a Lyapunov functional

$$
\begin{aligned}
V & =\sum_{k=1}^{n} v_{k}\left[\sum_{i=1}^{2} p_{i k}\left(S_{i}-S_{i}^{*}-S_{i}^{*} \ln \frac{S_{i}}{S_{i}^{*}}\right)\right. \\
& \left.+\int_{I_{k}^{*}}^{I_{k}} \frac{G_{k}(x)-G_{k}\left(I_{k}^{*}\right)}{G_{k}(x)} d x\right] .
\end{aligned}
$$

Differentiating $V$ along the solution of system (1), we obtain

$$
\begin{aligned}
\dot{V} & =\sum_{k=1}^{n} v_{k}\left\{p_{1 k}\left[\varphi\left(S_{1}\right)-a S_{1}\right]+p_{2 k}\left[a S_{1}-d_{2} S_{2}\right]\right. \\
& -m_{k} I_{k}-\frac{p_{1 k} S_{1}^{*}}{S_{1}}\left[\varphi\left(S_{1}\right)-\sum_{j=1}^{n} \beta_{1 j} S_{1} G_{j}\left(I_{j}\right)-a S_{1}\right] \\
& -\frac{p_{2 k} S_{2}^{*}}{S_{2}}\left[a S_{1}-\sum_{j=1}^{n} \beta_{2 j} S_{2} G_{j}\left(I_{j}\right)-d_{2} S_{2}\right] \\
& \left.-\frac{G_{k}\left(I_{k}^{*}\right)}{G_{k}\left(I_{k}\right)}\left[\sum_{i=1}^{2} p_{i k} \sum_{j=1}^{n} \beta_{i j} S_{i} G_{j}\left(I_{j}\right)-m_{k} I_{k}\right]\right\} \\
& =\sum_{k=1}^{n} v_{k}\left\{\varphi\left(S_{1}\right)\left(p_{1 k}-\frac{p_{1 k} S_{1}^{*}}{S_{1}}\right)\right. \\
& \left.-\frac{G_{k}\left(I_{k}^{*}\right)}{G_{k}\left(I_{k}\right)}\left[\sum_{i=1}^{2} p_{i k} \sum_{j=1}^{n} \beta_{i j} S_{i} G_{j}\left(I_{j}\right)\right]\right\} . \\
+ & d_{2} S_{2}^{*}\left(p_{2 k}-\frac{p_{2 k} S_{2}}{S_{2}^{*}}\right)+\sum_{i=1}^{2} p_{i k} \sum_{j=1}^{n} \beta_{i j} S_{i}^{*} G_{j}\left(I_{j}\right) \\
+ & \frac{m_{k} G_{k}\left(I_{k}^{*}\right) I_{k}}{G_{k}\left(I_{k}\right)}-m_{k} I_{k} \\
S_{1}^{*} & \left.\frac{p_{1 k} S_{1}}{S_{1}^{*} S_{1}}-\frac{p_{2 k} S_{1} S_{2}^{*}}{S_{2}}\right]
\end{aligned}
$$

From (7), we know that

$$
\begin{aligned}
\varphi\left(S_{1}^{*}\right)\left(p_{1 k}-\frac{p_{1 k} S_{1}^{*}}{S_{1}}\right) \\
=\left(\sum_{i=1}^{2} \sum_{j=1}^{n} \beta_{i j} S_{i}^{*} G_{j}\left(I_{j}^{*}\right)+d_{2} S_{2}^{*}\right)\left(p_{1 k}-\frac{p_{1 k} S_{1}^{*}}{S_{1}}\right) .
\end{aligned}
$$


It follows from (8), (9), and (19) that

$$
\begin{aligned}
\dot{V} & =\sum_{k=1}^{n} v_{k}\left\{\left[\varphi\left(S_{1}\right)-\varphi\left(S_{1}^{*}\right)\right]\left(p_{1 k}-\frac{p_{1 k} S_{1}^{*}}{S_{1}}\right)\right. \\
& +d_{2} S_{2}^{*}\left(p_{2 k}-\frac{p_{2 k} S_{2}}{S_{2}^{*}}\right)+\sum_{i=1}^{2} p_{i k} \beta_{i j} S_{i j}^{*} G_{j}\left(I_{j}^{*}\right) \\
& \cdot\left(\frac{G_{k}\left(I_{k}^{*}\right) I_{k}}{G_{k}\left(I_{k}\right) I_{k}^{*}}+\frac{G_{j}\left(I_{j}\right)}{G_{j}\left(I_{j}^{*}\right)}-\frac{I_{k}}{I_{k}^{*}}\right) \\
& +\sum_{j=1}^{n} \beta_{1 j} S_{1}^{*} G_{j}\left(I_{j}^{*}\right)\left(p_{1 k}-\frac{p_{1 k} S_{1}^{*}}{S_{1}}\right) \\
& +\left(d_{2} S_{2}^{*}+\sum_{j=1}^{n} \beta_{2 j} S_{2}^{*} G_{j}\left(I_{j}^{*}\right)\right) \\
& \cdot\left[2 p_{1 k}-\frac{p_{1 k} S_{1}^{*}}{S_{1}}-\frac{p_{1 k} S_{1}}{S_{1}^{*}}+\frac{p_{2 k} S_{1}}{S_{1}^{*}}-\frac{p_{2 k} S_{1} S_{2}^{*}}{S_{1}^{*} S_{2}}\right] \\
& \left.-\frac{G_{k}\left(I_{k}^{*}\right)}{G_{k}\left(I_{k}\right)}\left[\sum_{i=1}^{2} p_{i k} \sum_{j=1}^{n} \beta_{i j} S_{i} G_{j}\left(I_{j}\right)\right]\right\} .
\end{aligned}
$$

From (10), (15), and (16), we obtain

$$
\begin{aligned}
\dot{V} & \leq \sum_{k=1}^{n} v_{k}\left\{d_{2} S_{2}^{*}\left(p_{2 k}-\frac{p_{2 k} S_{2}}{S_{2}^{*}}\right)+\sum_{i=1}^{2} p_{i k} \beta_{i j} S_{i j}^{*} G_{j}\left(I_{j}^{*}\right)\right. \\
& +\sum_{j=1}^{n} \beta_{1 j} S_{1}^{*} G_{j}\left(I_{j}^{*}\right)\left(p_{1 k}-\frac{p_{1 k} S_{1}^{*}}{S_{1}}\right)+\left(d_{2} S_{2}^{*}\right. \\
& \left.+\sum_{j=1}^{n} \beta_{2 j} S_{2}^{*} G_{j}\left(I_{j}^{*}\right)\right)\left[2 p_{1 k}-\frac{p_{1 k} S_{1}^{*}}{S_{1}}-\frac{p_{1 k} S_{1}}{S_{1}^{*}}\right. \\
& \left.+\frac{p_{2 k} S_{1}}{S_{1}^{*}}-\frac{p_{2 k} S_{1} S_{2}^{*}}{S_{1}^{*} S_{2}}\right]-\frac{G_{k}\left(I_{k}^{*}\right)}{G_{k}\left(I_{k}\right)}\left[\sum_{i=1}^{2} p_{i k}\right. \\
& \left.\left.\cdot \sum_{j=1}^{n} \beta_{i j} S_{i} G_{j}\left(I_{j}\right)\right]\right\}=\sum_{k=1}^{n} v_{k}\left\{d _ { 2 } S _ { 2 } ^ { * } \left(p_{2 k}+2 p_{1 k}\right.\right. \\
& \left.-\frac{p_{1 k} S_{1}^{*}}{S_{1}}-\frac{p_{1 k} S_{1}}{S_{1}^{*}}+\frac{p_{2 k} S_{1}}{S_{1}^{*}}-\frac{p_{2 k} S_{1} S_{2}^{*}}{S_{1}^{*} S_{2}}-\frac{p_{2 k} S_{2}}{S_{2}^{*}}\right) \\
& +\sum_{j=1}^{n} \beta_{2 j} S_{2}^{*} G_{j}\left(I_{j}^{*}\right) \\
& +\left[2 p_{1 k}+p_{2 k}-\frac{p_{1 k} S_{1}^{*}}{S_{1}}-\frac{p_{1 k} S_{1}}{S_{1}^{*}}+\frac{p_{2 k} S_{1}}{S_{1}^{*}}-\frac{p_{2 k} S_{1} S_{2}^{*}}{S_{1}^{*} S_{2}}\right] \\
& +\sum_{j=1}^{n} \beta_{1 j} S_{1}^{*} G_{j}\left(I_{j}^{*}\right)\left(2 p_{1 k}-\frac{p_{1 k} S_{1}^{*}}{S_{1}}\right)
\end{aligned}
$$

$$
\begin{aligned}
& \left.-\frac{G_{k}\left(I_{k}^{*}\right)}{G_{k}\left(I_{k}\right)}\left[\sum_{i=1}^{2} p_{i k} \sum_{j=1}^{n} \beta_{i j} S_{i} G_{j}\left(I_{j}\right)\right]\right\}=-\sum_{k=1}^{n} v_{k}\left(p_{1 k}\right. \\
& \left.-p_{2 k}\right)\left(d_{2} S_{2}^{*}+\sum_{j=1}^{n} \beta_{2 j} S_{2}^{*} G_{j}\left(I_{j}^{*}\right)\right)\left(\frac{S_{1}^{*}}{S_{1}}+\frac{S_{1}}{S_{1}^{*}}\right) \\
& +\sum_{k=1}^{n} v_{k}\left\{d _ { 2 } S _ { 2 } ^ { * } \left[p_{2 k}+2 p_{1 k}\right.\right. \\
& \left.-p_{2 k}\left(\frac{S_{1}^{*}}{S_{1}}+\frac{S_{1} S_{2}^{*}}{S_{1}^{*} S_{2}}+\frac{S_{2}}{S_{2}^{*}}\right)\right]+\sum_{j=1}^{n} \beta_{2 j} S_{2}^{*} G_{j}\left(I_{j}^{*}\right)\left[2 p_{1 k}\right. \\
& \left.+p_{2 k}-p_{2 k}\left(\frac{S_{1}^{*}}{S_{1}}+\frac{S_{1} S_{2}^{*}}{S_{1}^{*} S_{2}}+\frac{S_{2} G_{k}\left(I_{k}^{*}\right) G_{j}\left(I_{j}\right)}{S_{2}^{*} G_{k}\left(I_{k}\right) G_{j}\left(I_{j}^{*}\right)}\right)\right] \\
& +\sum_{j=1}^{n} \beta_{1 j} S_{1}^{*} G_{j}\left(I_{j}^{*}\right) \\
& \left..\left[2 p_{1 k}-p_{1 k}\left(\frac{S_{1}^{*}}{S_{1}}+\frac{S_{1} G_{k}\left(I_{k}^{*}\right) G_{j}\left(I_{j}\right)}{S_{1}^{*} G_{k}\left(I_{k}\right) G_{j}\left(I_{j}^{*}\right)}\right)\right]\right\}=: B_{1} .
\end{aligned}
$$

By $\sum_{k=1}^{n} v_{k}\left(p_{1 k}-p_{2 k}\right) \geq 0$ and the arithmetic-geometric mean, we easily see that

$$
\begin{aligned}
B_{1} \leq & -\sum_{k=1}^{n} v_{k}\left(2 p_{1 k}-2 p_{2 k}\right)\left(d_{2} S_{2}^{*}\right. \\
& \left.+\sum_{j=1}^{n} \beta_{2 j} S_{2}^{*} G_{j}\left(I_{j}^{*}\right)\right)+\sum_{k=1}^{n} v_{k}\left\{d _ { 2 } S _ { 2 } ^ { * } \left[p_{2 k}+2 p_{1 k}\right.\right. \\
& \left.-3 p_{2 k}\right]+\sum_{j=1}^{n} \beta_{2 j} S_{2}^{*} G_{j}\left(I_{j}^{*}\right) \\
& .\left[2 p_{1 k}+p_{2 k}-3 p_{2 k}\left[\frac{G_{k}\left(I_{k}^{*}\right) G_{j}\left(I_{j}\right)}{G_{k}\left(I_{k}\right) G_{j}\left(I_{j}^{*}\right)}\right]^{1 / 3}\right] \\
& +\sum_{j=1}^{n} \beta_{1 j} S_{1}^{*} G_{j}\left(I_{j}^{*}\right) \\
& \left.\cdot\left[2 p_{1 k}-2 p_{1 k}\left[\frac{G_{k}\left(I_{k}^{*}\right) G_{j}\left(I_{j}\right)}{G_{k}\left(I_{k}\right) G_{j}\left(I_{j}^{*}\right)}\right]^{1 / 2}\right]\right\} \\
& =\sum_{k=1}^{n} v_{k}\left\{\sum _ { j = 1 } ^ { n } \beta _ { 2 j } S _ { 2 } ^ { * } G _ { j } ( I _ { j } ^ { * } ) \left[3 p_{2 k}\right.\right.
\end{aligned}
$$




$$
\begin{aligned}
& \left.-3 p_{2 k}\left[\frac{G_{k}\left(I_{k}^{*}\right) G_{j}\left(I_{j}\right)}{G_{k}\left(I_{k}\right) G_{j}\left(I_{j}^{*}\right)}\right]^{1 / 3}\right]+\sum_{j=1}^{n} \beta_{1 j} S_{1}^{*} G_{j}\left(I_{j}^{*}\right) \\
& \left.\cdot\left[2 p_{1 k}-2 p_{1 k}\left[\frac{G_{k}\left(I_{k}^{*}\right) G_{j}\left(I_{j}\right)}{G_{k}\left(I_{k}\right) G_{j}\left(I_{j}^{*}\right)}\right]^{1 / 2}\right]\right\}=: B_{2} .
\end{aligned}
$$

We can rewrite $B_{2}$ as

$$
\begin{aligned}
B_{2} & =\sum_{k=1}^{n} v_{k}\left\{3 \sum_{j=1}^{n} p_{2 k} \beta_{2 j} S_{2}^{*} G_{j}\left(I_{j}^{*}\right)\right. \\
& .\left[1-\left[\frac{G_{k}\left(I_{k}^{*}\right) G_{j}\left(I_{j}\right)}{G_{k}\left(I_{k}\right) G_{j}\left(I_{j}^{*}\right)}\right]^{1 / 3}+\ln \left[\frac{G_{k}\left(I_{k}^{*}\right) G_{j}\left(I_{j}\right)}{G_{k}\left(I_{k}\right) G_{j}\left(I_{j}^{*}\right)}\right]^{1 / 3}\right] \\
& +2 \sum_{j=1}^{n} p_{1 k} \beta_{1 j} S_{1}^{*} G_{j}\left(I_{j}^{*}\right) \\
& .\left[1-\left[\frac{G_{k}\left(I_{k}^{*}\right) G_{j}\left(I_{j}\right)}{G_{k}\left(I_{k}\right) G_{j}\left(I_{j}^{*}\right)}\right]^{1 / 2}+\ln \left[\frac{G_{k}\left(I_{k}^{*}\right) G_{j}\left(I_{j}\right)}{G_{k}\left(I_{k}\right) G_{j}\left(I_{j}^{*}\right)}\right]^{1 / 2}\right] \\
& \left.-\sum_{i}^{2} \sum_{j=1}^{n} p_{i k} \beta_{i j} S_{i}^{*} G_{j}\left(I_{j}^{*}\right) \ln \left[\frac{G_{k}\left(I_{k}^{*}\right) G_{j}\left(I_{j}\right)}{G_{k}\left(I_{k}\right) G_{j}\left(I_{j}^{*}\right)}\right]\right\} .
\end{aligned}
$$

Using the fact that $1-x+\ln x \leq 0$, where equality holds if only if $x=1$, we obtain

$$
\begin{aligned}
& B_{2} \\
& \leq-\sum_{k=1}^{n} v_{k} \sum_{i}^{2} \sum_{j=1}^{n} p_{i k} \beta_{i j} S_{i}^{*} G_{j}\left(I_{j}^{*}\right) \ln \left[\frac{G_{k}\left(I_{k}^{*}\right) G_{j}\left(I_{j}\right)}{G_{k}\left(I_{k}\right) G_{j}\left(I_{j}^{*}\right)}\right] \\
& =\sum_{k, j=1}^{n} v_{k} \bar{\beta}_{k j} \ln \frac{G_{k}\left(I_{k}\right) G_{j}\left(I_{j}^{*}\right)}{G_{k}\left(I_{k}^{*}\right) G_{j}\left(I_{j}\right)} .
\end{aligned}
$$

In the following, we will show that

$$
\bar{H}_{n}:=\sum_{k, j=1}^{n} v_{k} \bar{\beta}_{k j} \ln \frac{G_{k}\left(I_{k}\right) G_{j}\left(I_{j}^{*}\right)}{G_{k}\left(I_{k}^{*}\right) G_{j}\left(I_{j}\right)} \equiv 0 .
$$

We first give the proof of (25) for $n=2$, which would give a reader the basic yet clear ideas without being hidden by the complexity of terms caused by larger values of $n$. When $n=2$, we have $\bar{H}_{2}=\sum_{k, j=1}^{2} v_{k} \bar{\beta}_{k j} \ln \left(G_{k}\left(I_{k}\right) G_{j}\left(I_{j}^{*}\right) / G_{k}\left(I_{k}^{*}\right) G_{j}\left(I_{j}\right)\right)$.
Formula (13) gives $v_{1}=\bar{\beta}_{21}$ and $v_{2}=\bar{\beta}_{12}$ in this case. Expanding $\bar{H}_{2}$ yields

$$
\begin{aligned}
\bar{H}_{2} & \\
= & \bar{\beta}_{21} \bar{\beta}_{11} \ln \frac{G_{1}\left(I_{1}\right) G_{1}\left(I_{1}^{*}\right)}{G_{1}\left(I_{1}^{*}\right) G_{1}\left(I_{1}\right)} \\
& +\bar{\beta}_{12} \bar{\beta}_{22} \ln \frac{G_{2}\left(I_{2}\right) G_{2}\left(I_{2}^{*}\right)}{G_{2}\left(I_{2}^{*}\right) G_{2}\left(I_{2}\right)} \\
& +\bar{\beta}_{21} \bar{\beta}_{12} \ln \frac{G_{1}\left(I_{1}\right) G_{2}\left(I_{2}^{*}\right)}{G_{1}\left(I_{1}^{*}\right) G_{2}\left(I_{2}\right)} \\
& +\bar{\beta}_{12} \bar{\beta}_{21} \ln \frac{G_{2}\left(I_{2}\right) G_{1}\left(I_{1}^{*}\right)}{G_{2}\left(I_{2}^{*}\right) G_{1}\left(I_{1}\right)} \\
= & \bar{\beta}_{12} \bar{\beta}_{21}\left[\ln \frac{G_{1}\left(I_{1}\right) G_{2}\left(I_{2}^{*}\right)}{G_{1}\left(I_{1}^{*}\right) G_{2}\left(I_{2}\right)}+\ln \frac{G_{2}\left(I_{2}\right) G_{1}\left(I_{1}^{*}\right)}{G_{2}\left(I_{2}^{*}\right) G_{1}\left(I_{1}\right)}\right] \\
= & 0 .
\end{aligned}
$$

For more general $n$, by a similar argument as in the proof of $\sum_{k, j=1}^{n} v_{k} \bar{\beta}_{k j} \ln \left(E_{k}^{*} E_{j} / E_{k} E_{j}^{*}\right) \equiv 0$ in [16], we obtain $\sum_{k, j=1}^{n} v_{k} \bar{\beta}_{k j} \ln \left(G_{k}\left(I_{k}\right) G_{j}\left(I_{j}^{*}\right) / G_{k}\left(I_{k}^{*}\right) G_{j}\left(I_{\mathrm{j}}\right)\right) \equiv 0$.

From (21), (22), and (24), we see that if $\dot{V}=0$, then

$$
S_{i}=S_{i}^{*}, \quad i=1,2 .
$$

If (27) holds, it follows from (1) that

$$
\begin{aligned}
& 0=\varphi\left(S_{1}^{*}\right)-\sum_{j=1}^{n} \beta_{1 j} S_{1}^{*} G_{j}\left(I_{j}\right)-a S_{1}^{*}, \\
& 0=a S_{1}^{*}-\sum_{j=1}^{n} \beta_{2 j} S_{2}^{*} G_{j}\left(I_{j}\right)-d_{2} S_{2}^{*} .
\end{aligned}
$$

Then, we obtain that

$$
\begin{array}{r}
\dot{I_{k}}=p_{1 k}\left(\varphi\left(S_{1}^{*}\right)-a S_{1}^{*}\right)+p_{2 k}\left(a S_{1}^{*}-d_{2} S_{2}^{*}\right)-m_{k} I_{k}, \\
k=1,2, \ldots, n .
\end{array}
$$

This implies that

$$
\begin{aligned}
\lim _{t \rightarrow+\infty} I_{k} & =\frac{p_{1 k}\left(\varphi\left(S_{1}^{*}\right)-a S_{1}^{*}\right)+p_{2 k}\left(a S_{1}^{*}-d_{2} S_{2}^{*}\right)}{m_{k}} \\
& =I_{k}^{*} .
\end{aligned}
$$

By the characteristics of $V$, we obtain the idea that the largest invariant subset of the set where $\dot{V}=0$ is the singleton $\left\{P^{*}\right\}$. By LaSalle's Invariance Principle, $P^{*}$ is globally asymptotically stable for $R_{0}>1$. This completes the proof. 

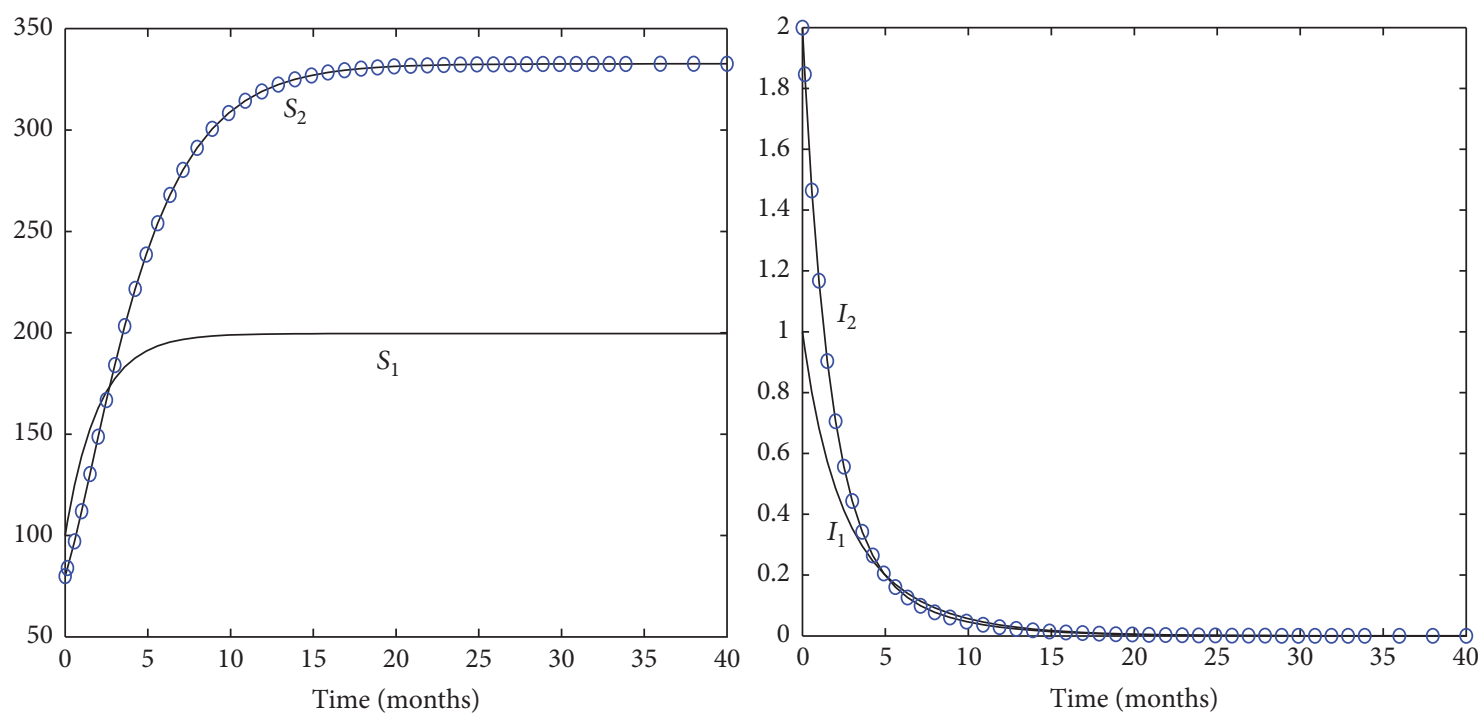

Figure 1: Dynamical behavior of system (31) with parameter values in (32) and Case $1 . R_{0} \approx 0.55$. The initial conditions are $S_{1}(0)=100$, $S_{2}(0)=80, I_{1}(0)=1$, and $I_{2}(0)=2$. We see that the disease dies out in both subgroups.

\section{Numerical Examples}

In the section, numerical simulations are presented to support and complement the theoretical findings. We consider the following model:

$$
\begin{aligned}
& \dot{S_{1}}=\varphi\left(S_{1}\right)-\sum_{j=1}^{2} \beta_{1 j} S_{1} G_{j}\left(I_{j}\right)-a S_{1}, \\
& \dot{S_{2}}=a S_{1}-\sum_{j=1}^{2} \beta_{2 j} S_{2} G_{j}\left(I_{j}\right)-d_{2} S_{2}, \\
& \dot{I_{1}}=p_{11} \sum_{j=1}^{2} \beta_{1 j} S_{1} G_{j}\left(I_{j}\right)+p_{21} \sum_{j=1}^{2} \beta_{2 j} S_{2} G_{j}\left(I_{j}\right)-m_{1} I_{1}, \\
& \dot{I}_{2}=p_{12} \sum_{j=1}^{2} \beta_{1 j} S_{1} G_{j}\left(I_{j}\right)+p_{22} \sum_{j=1}^{2} \beta_{2 j} S_{2} G_{j}\left(I_{j}\right)-m_{2} I_{2},
\end{aligned}
$$

where $G_{j}\left(I_{j}\right)=I_{j} /\left(1+\alpha_{j} I_{j}\right)$. Clearly, (A1) and (A2) hold. We fix the parameters as follows:

$$
\begin{aligned}
b & =100, \\
d_{1} & =0.001, \\
d_{2} & =0.3, \\
a & =0.5, \\
\alpha_{1} & =\alpha_{2}=0.1, \\
m_{1} & =0.5, \\
m_{2} & =0.6,
\end{aligned}
$$

$$
\begin{aligned}
& p_{11}=p_{22}=0.6 \\
& p_{12}=p_{21}=0.4 .
\end{aligned}
$$

Then, we have $P_{0} \approx(199.6008,332.6680,0,0)$.

Case 1. If $\beta_{11}=\beta_{21}=0.001, \beta_{12}=\beta_{22}=0.0001$, then we obtain $R_{0} \approx 0.55$. By Theorem 1 , the disease dies out in both subgroups. Numerical simulation illustrates this fact (see Figure 1).

Case 2. If $\beta_{11}=\beta_{21}=0.01, \beta_{12}=\beta_{22}=0.001$, then we have $R_{0} \approx 5.53, P^{*} \approx(171.0530,222.9491,32.0764,28.1767)$, $v_{1}=\bar{\beta}_{21}=\sum_{i=1}^{2} p_{i 2} \beta_{i 1} S_{i}^{*} G_{1}\left(I_{1}^{*}\right) \approx 154.06, v_{2}=\bar{\beta}_{12}=$ $\sum_{i=1}^{2} p_{i 1} \beta_{i 2} S_{i}^{*} G_{2}\left(I_{2}^{*}\right) \approx 73.81$, and $\sum_{k=1}^{2} v_{k}\left(p_{1 k}-p_{2 k}\right) \approx$ 16.05. By Theorem 2 , the disease persists in both subgroups. Numerical simulation illustrates this fact (see Figure 2).

\section{Conclusions}

A differential infectivity epidemic model with stage structure has been used to describe the spreading of such a disease. We have focused on the theoretical analysis of the equilibriums. Using a graph-theoretic approach to the method of Lyapunov functions, we have proved the global stability of the endemic equilibrium. We have established uniform persistence and the sharp threshold. The work has potential extensions and improvements, which remains to be discussed in the future.

\section{Competing Interests}

The author declares that there are no competing interests. 

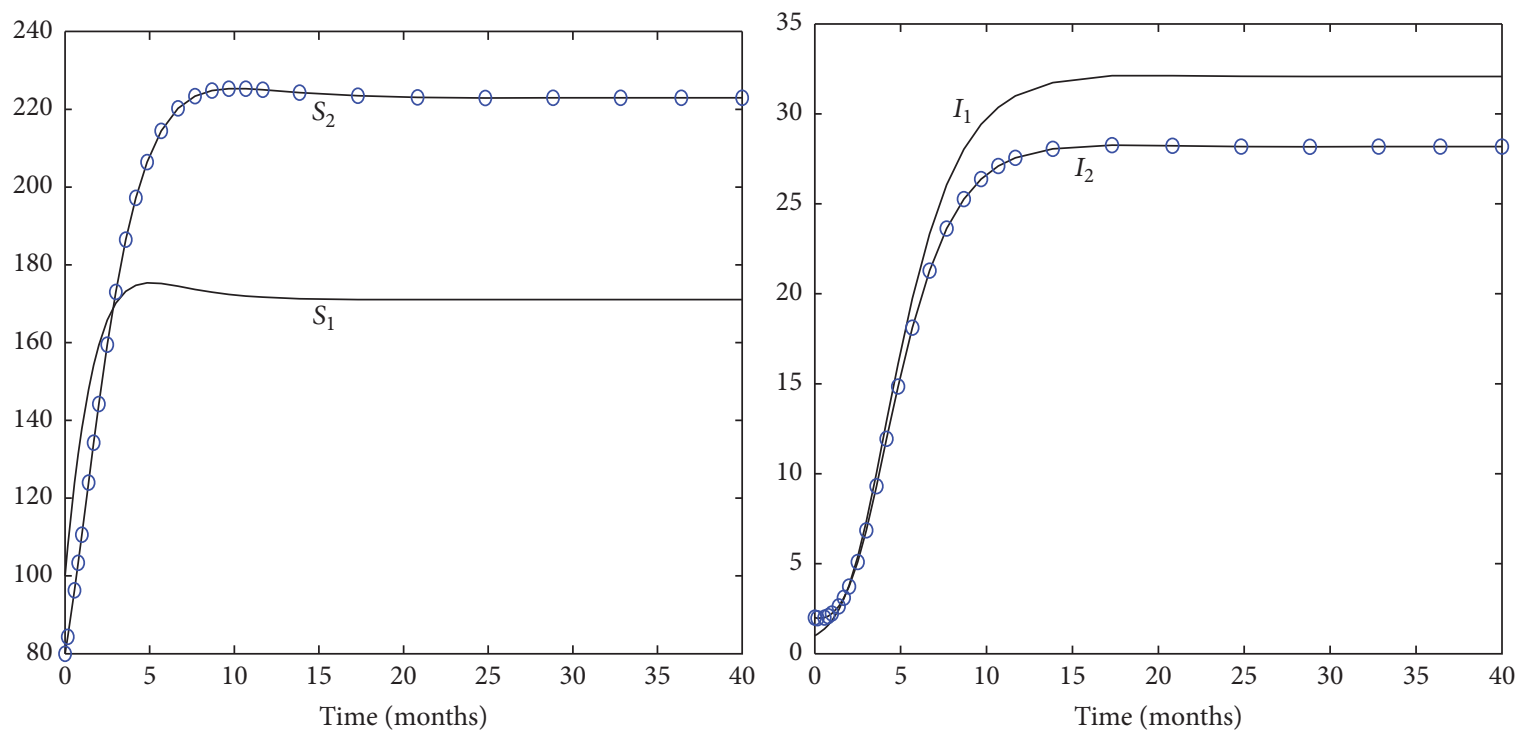

FIGURE 2: Dynamical behavior of system (31) with parameter values in (32) and Case $1 . R_{0} \approx 5.53$. The initial conditions are $S_{1}(0)=100$, $S_{2}(0)=80, I_{1}(0)=1$, and $I_{2}(0)=2$. We see that the disease persists in both subgroups.

\section{Acknowledgments}

This work was supported by Key Laboratory of Statistical Information Technology and Data Mining, State Statistics Bureau (SDL201601).

\section{References}

[1] J. M. Hyman, J. Li, and E. A. Stanley, "The differential infectivity and staged progression models for the transmission of HIV," Mathematical Biosciences, vol. 155, no. 2, pp. 77-109, 1999.

[2] Z. Ma, J. Liu, and J. Li, "Stability analysis for differential infectivity epidemic models," Nonlinear Analysis. Real World Applications, vol. 4, no. 5, pp. 841-856, 2003.

[3] A. Alexanderian, M. K. Gobbert, K. R. Fister, H. Gaff, S. Lenhart, and E. Schaefer, "An age-structured model for the spread of epidemic cholera: analysis and simulation," Nonlinear Analysis: Real World Applications, vol. 12, no. 6, pp. 3483-3498, 2011.

[4] Y. Liu, S. Gao, and Y. Luo, "Impulsive epidemic model with differential susceptibility and stage structure," Applied Mathematical Modelling, vol. 36, no. 1, pp. 370-378, 2012.

[5] X.-B. Zhang, H.-F. Huo, X.-K. Sun, and Q. Fu, "The differential susceptibility SIR epidemic model with stage structure and pulse vaccination," Nonlinear Analysis. Real World Applications, vol. 11, no. 4, pp. 2634-2646, 2010.

[6] X. Shi, J. Cui, and X. Zhou, "Stability and Hopf bifurcation analysis of an eco-epidemic model with a stage structure," Nonlinear Analysis. Theory, Methods \& Applications, vol. 74, no. 4, pp. 1088-1106, 2011.

[7] C. Wu and P. Weng, "Stability analysis of a SIS model with stage structured and distributed maturation delay," Nonlinear Analysis: Theory, Methods \& Applications, vol. 71, no. 12, pp. e892-e901, 2009.

[8] H. Inaba, "Endemic threshold results in an age-durationstructured population model for HIV infection," Mathematical Biosciences, vol. 201, no. 1-2, pp. 15-47, 2006.
[9] Z. Feng, W. Huang, and C. Castillo-Chavez, "Global behavior of a multi-group SIS epidemic model with age structure," Journal of Differential Equations, vol. 218, no. 2, pp. 292-324, 2005.

[10] B. Tian, Y. Jin, S. Zhong, and N. Chen, "Global stability of an epidemic model with stage structure and nonlinear incidence rates in a heterogeneous host population," Advances in Difference Equations, vol. 2015, article 260, 2015.

[11] H. Guo, M. Y. Li, and Z. Shuai, "Global stability of the endemic equilibrium of multigroup SIR epidemic models," Canadian Applied Mathematics Quarterly, vol. 14, no. 3, pp. 259-284, 2006.

[12] R. Sun and J. Shi, "Global stability of multigroup epidemic model with group mixing and nonlinear incidence rates," Applied Mathematics and Computation, vol. 218, no. 2, pp. 280286, 2011.

[13] Z. Yuan and L. Wang, "Global stability of epidemiological models with group mixing and nonlinear incidence rates," Nonlinear Analysis. Real World Applications, vol. 11, no. 2, pp. 995-1004, 2010.

[14] T. Kuniya, "Global stability analysis with a discretization approach for an age-structured multigroup SIR epidemic model," Nonlinear Analysis: Real World Applications, vol. 12, no. 5, pp. 2640-2655, 2011.

[15] Z. Yuan and X. Zou, "Global threshold property in an epidemic model for disease with latency spreading in a heterogeneous host population," Nonlinear Analysis. Real World Applications, vol. 11, no. 5, pp. 3479-3490, 2010.

[16] R. Sun, "Global stability of the endemic equilibrium of multigroup SIR models with nonlinear incidence," Computers \& Mathematics with Applications, vol. 60, no. 8, pp. 2286-2291, 2010.

[17] M. Y. Li, Z. Shuai, and C. Wang, "Global stability of multigroup epidemic models with distributed delays," Journal of Mathematical Analysis and Applications, vol. 361, no. 1, pp. 3847, 2010.

[18] H. Shu, D. Fan, and J. Wei, "Global stability of multi-group SEIR epidemic models with distributed delays and nonlinear transmission," Nonlinear Analysis: Real World Applications, vol. 13, no. 4, pp. 1581-1592, 2012. 
[19] D. Ding and X. Ding, "Global stability of multi-group vaccination epidemic models with delays," Nonlinear Analysis. Real World Applications, vol. 12, no. 4, pp. 1991-1997, 2011.

[20] H. Chen and J. Sun, "Global stability of delay multigroup epidemic models with group mixing and nonlinear incidence rates," Applied Mathematics and Computation, vol. 218, no. 8, pp. 4391-4400, 2011.

[21] C. Zhang, W. Li, and K. Wang, "Graph theory-based approach for stability analysis of stochastic coupled systems with Lévy noise on networks," IEEE Transactions on Neural Networks and Learning Systems, vol. 26, no. 8, pp. 1698-1709, 2015.

[22] C. Zhang, W. Li, and K. Wang, "Graph-theoretic approach to stability of multi-group models with dispersal," Discrete and Continuous Dynamical Systems Series B, vol. 20, no. 1, pp. 259280, 2015.

[23] H. I. Freedman, M. X. Tang, and S. G. Ruan, "Uniform persistence and flows near a closed positively invariant set," Journal of Dynamics and Differential Equations, vol. 6, no. 4, pp. 583-600, 1994.

[24] M. Y. Li, J. R. Graef, L. Wang, and J. Karsai, "Global dynamics of a SEIR model with varying total population size," Mathematical Biosciences, vol. 160, no. 2, pp. 191-213, 1999.

[25] N. P. Bhatia and G. P. Szegö, Dynamical Systems: Stability Theory and Applications, Lecture Notes in Mathematics, No. 35, Springer, New York, NY, USA, 1967.

[26] H. L. Smith and P. Waltman, The Theory of the Chemostat: Dynamics of Microbial Competition, Cambridge University Press, Cambridge, UK, 1995. 


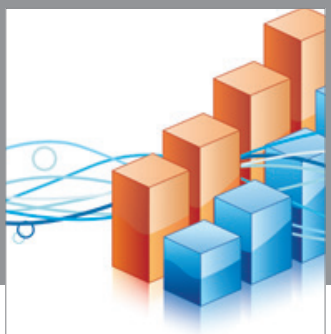

Advances in

Operations Research

vatem alat4

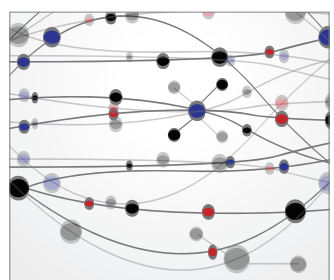

\section{The Scientific} World Journal
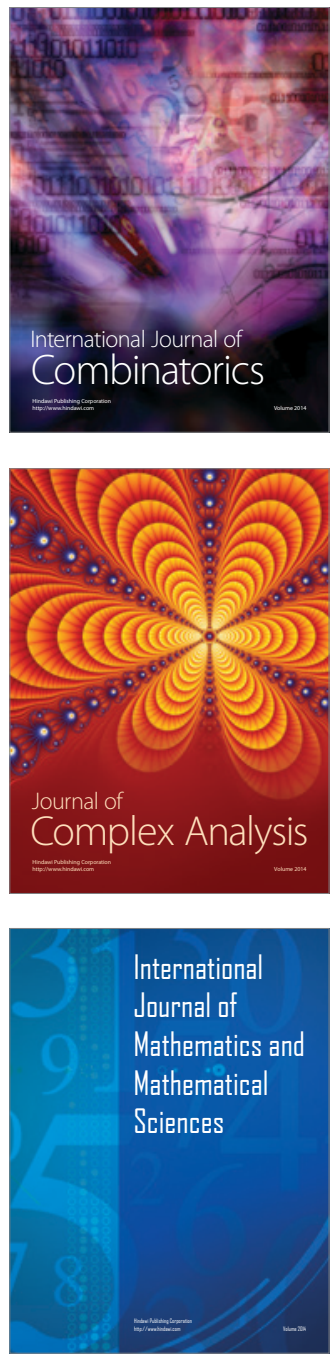
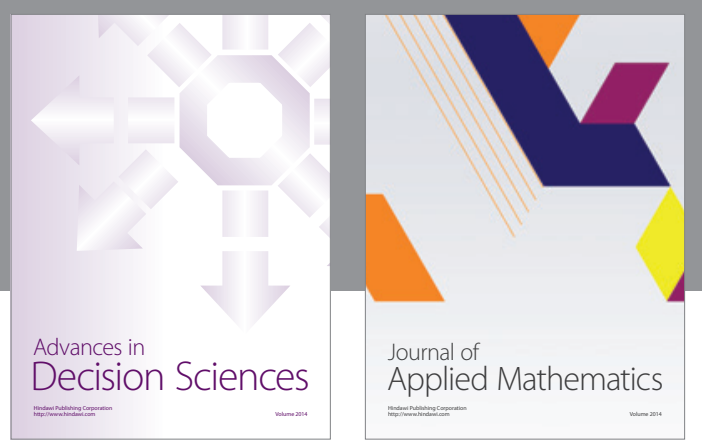

Algebra

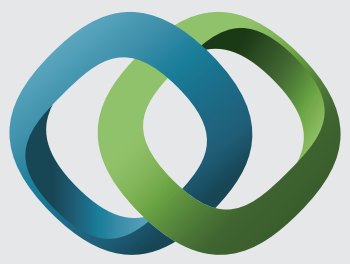

\section{Hindawi}

Submit your manuscripts at

http://www.hindawi.com
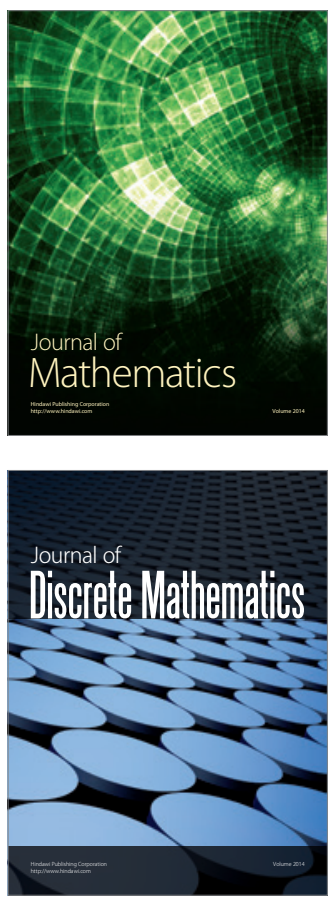

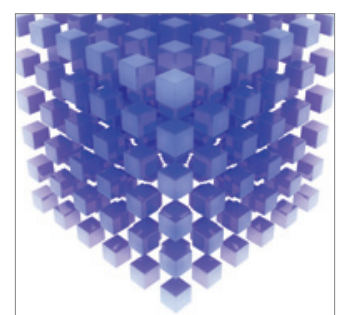

Mathematical Problems in Engineering
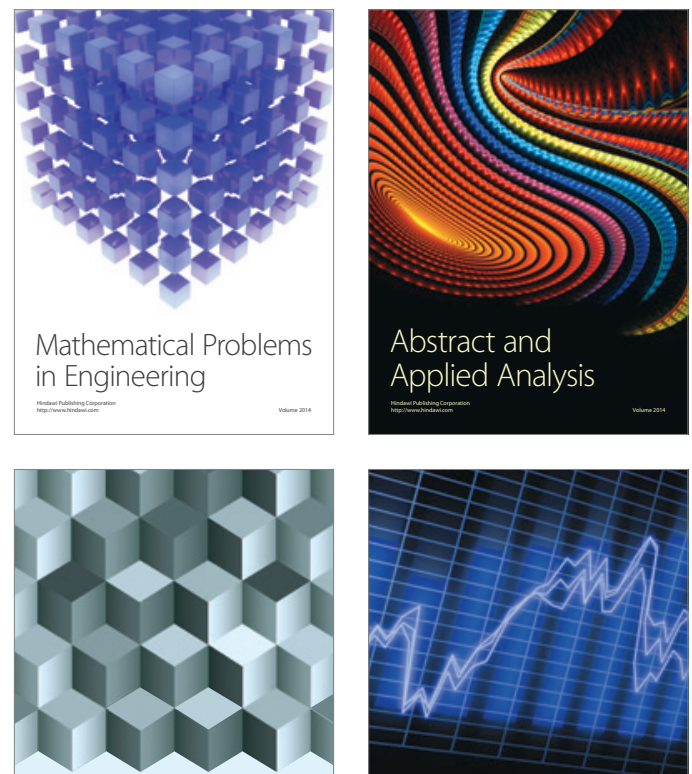

Journal of

Function Spaces

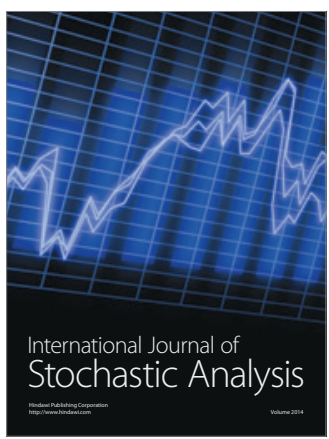

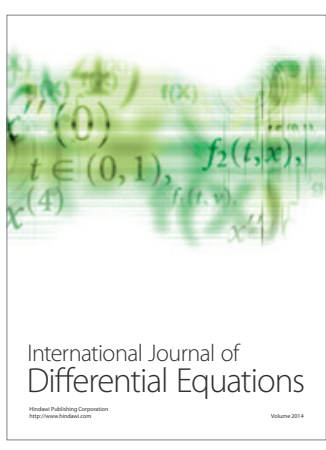
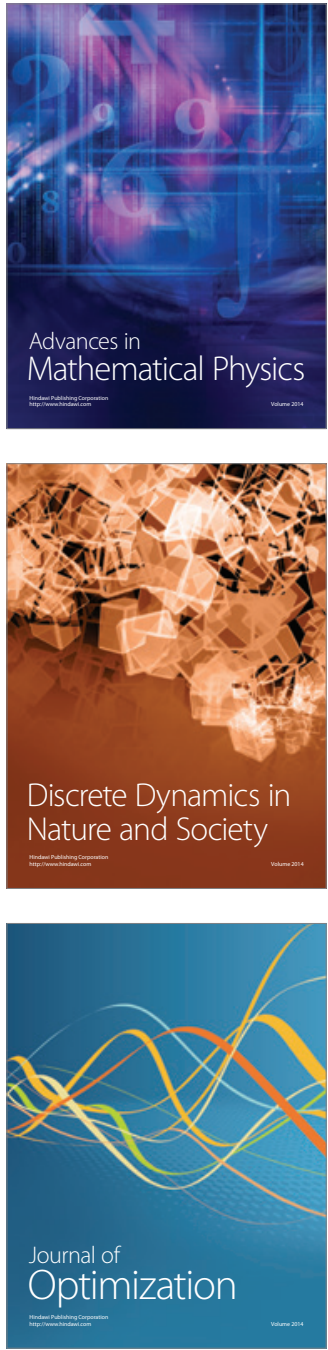